\title{
Semiclassical and Quantum-Mechanical Formalism Applied in Calculating the Emission Intensity of the Atomic Hydrogen
}

\author{
Stanisław Olszewski \\ Institute of Physical Chemistry, Polish Academy of Sciences, Warsaw, Poland \\ Email: olsz@ichf.edu.pl
}

Received 26 April 2016; accepted 28 May 2016; published 31 May 2016

Copyright @ 2016 by author and Scientific Research Publishing Inc.

This work is licensed under the Creative Commons Attribution International License (CC BY).

http://creativecommons.org/licenses/by/4.0/

(c) () Open Access

\section{Abstract}

The energy spectrum of the hydrogen atom has been applied in calculating the time rate of energy transitions between the quantum states of the atom. The formal basis of the approach has been provided by the quantum properties of energy and time deduced from the Joule-Lenz law. The rates of the energy transitions obtained in this way were compared with the quantum-mechanical probabilities of transitions calculated earlier by Bethe and Condon and Shortley for the same pairs of the quantum states.

\section{Keywords}

Time and Intensity of Electron Transitions in the Atomic Hydrogen, Semiclassical Emission Intensities Compared with the Quantum-Mechanical Transition Probabilities

\section{Introduction}

The intensity of the electron transitions between quantum states in an atom met since a long time an important conceptual difficulty. Since the states are separated by indivisible quanta of energy, the problem of a gradual fulfillment of the energy gaps characterized by these quanta loses its sense and only a probabilistic approach concerning a whole of the quantum ensemble represented by a set of atoms should be considered. Such kind of the statistical reasoning has been applied to quantum states already in the framework of the old quantum theory [1]-[3], nevertheless the effective calculations of transition probabilities were provided, in general, only by the formalism of quantum mechanics [4] [5].

In practice, an application of such a quantum-mechanical background seems to be a rather tedious task, mainly because of a possibly accurate knowledge of the wave functions necessary to calculations. But in general the 
appropriate wave functions are difficult to assess, and remain much less accurate than the energies. This situation made it desirable to obtain a coupling between the energy quanta and the time intervals associated with the transitions which—intuitionally—should be represented by some finite amounts of time.

Fortunately such a coupling could be provided in effect of an analysis of single electron transitions in a quantum system done with the aid of the Joule-Lenz law. We assume that both the energy amount, and the interval of time entering this law, are characteristic for the electron transition. In effect a reference between the energy and time is defined by 1) the electric resistance connected with transition and 2) the electron current present in the system [6]-[8]. The current, on its side, is represented by the periodic one-electron motion in the system, a property which is well-known, for example, in the case of the electron circulation performed incessibly in the Bohr hydrogen atom. On the other hand, the resistance is obtained in effect of the presence of a potential difference connected with the energy change between two quantum levels.

When the electric resistance and current are combined together according to the Joule-Lenz law, we obtain readily a coupling between the energy change and that of time; see e.g. [9]. In considering the emission spectra we assume that the excited states of the atoms are approximately in thermal equilibrium, so the number of states in any given atom is proportional to the Boltzmann factor [10]. In this case the intensity of a spectral linewhich we assume is defined by the ratio of the energy interval $\Delta E$ to the time interval $\Delta t$ connected with transition - can be considered as proportional to the transition probability calculated with the aid of the quantum-mechnical formalism [10]. In order to check this property a spectrum of $n^{\prime} p-n^{\prime \prime} s$ transitions, which satisfy the relation

$$
n^{\prime}>n^{\prime \prime},
$$

and both $n^{\prime}$ and $n^{\prime \prime}$ are the low-lying quantum states of the hydrogen atom, has been studied [8]. The problem of the proportionality coefficient has been eliminated by considering a pattern of the ratios of intensities and that of probabilities corresponding to different pairs of the electron transitions. In general the pattern of semiclassical intensities is found to fit rather well to that obtained for the quantum-mechanical probabilities calculated for the same levels [8].

Certainly the selection rules for electron transitions, especially those dictated by the quanta of angular momentum belonging to $n^{\prime}$ and $n$, could not be included by the semiclassical theory. This raised the problem how the angular momentum parameters, for example different than those applied in [8], can influence the relation between the semiclassical intensities and quantum-mechanical probability results. To answer this point the present paper examines the ratios obtained for a spectrum of $n^{\prime} d-n^{\prime \prime} p$ transitions together with $n^{\prime} f-n^{\prime \prime} d$ and $n^{\prime} g-n^{\prime \prime} f$. In any of these cases the relation of (1) is preserved. This means that we referred the above transitions to those where the state $n^{\prime}$ of a higher energy is associated systematically with the state $l^{\prime}$ of a higher angular momentum; evidently the states $n^{\prime \prime}$ of the lower energy refer to those of the lower angular momentum $l^{\prime \prime}$.

But in spectroscopy also an opposite emission than from $n^{\prime} l^{\prime}$ to $n^{\prime \prime} l^{\prime \prime}$ in which

$$
l^{\prime}>l^{\prime \prime}
$$

can take place. To obtain an insight into the case of

$$
l^{\prime}<l^{\prime \prime},
$$

say by $l^{\prime}=0$ and $l^{\prime \prime}=1$, the $n^{\prime} s-n^{\prime \prime} p\left(n^{\prime}>n^{\prime \prime}\right)$ transitions have been also studied in the present paper. It is demonstrated that the accuracy of agreement obtained between the results of the semiclassical and quantum-mechanical formalisms depends on the assumption whether the case of (2) or (3) is taken into account.

\section{Basic Formulae}

An outline of the formulae which are of use in a semiclassical theory of the energy emission in the atom has been done before [8]. A general aim of the former and present paper was to provide a formalism which allowed us - in the first step - to calculate the emission intensity of individual spectral lines in the hydrogen atom with the aid of the energy quanta and time quanta connected with the energy emission. Since the time quanta can be next represented by the energy quanta (see [6]-[8]), the emission intensity is in fact expressed solely with the aid of the electron transition energies in the atom. The intensity ratios of different spectral lines obtained in this way can be compared with the quantum-mechanical ratios of transition probabilities characteristic for these lines. 
The fundamental energy quanta are

$$
\Delta E_{n}=E_{n+1}-E_{n}=f\left[\frac{1}{n^{2}}-\frac{1}{(n+1)^{2}}\right]
$$

where

$$
f=\frac{m_{e} e^{4}}{2 \hbar^{2}}
$$

( $m_{e}$ is the electron mass) and

$$
n=1,2,3, \cdots,
$$

label the quantum states of the hydrogen atom. The number $n=1$ represents the ground atomic state.

Let us note that (4) label the energy distances between the neighbouring states. But beyond of (4) the energy quanta between more distant states than neighbouring ones can also enter the calculations. In a particular case of the present paper the energy quanta which are of use become special cases of the formula

$$
\Delta E_{p-q}=E_{p}-E_{q}=f\left(\frac{1}{q^{2}}-\frac{1}{p^{2}}\right)
$$

namely

$$
(p, q)=(5,2),(6,2) \text { and }(6,3) \text {. }
$$

The (8) give respectively for (7):

$$
\begin{aligned}
& \Delta E_{5-2}=f \frac{21}{100}, \\
& \Delta E_{6-2}=f \frac{2}{9}, \\
& \Delta E_{6-3}=f \frac{1}{12} .
\end{aligned}
$$

A fundamental relation does exist between $\Delta E_{n}$ and $\Delta t_{n}$ where

$$
\Delta t_{n}=t_{n+1}-t_{n} .
$$

The relation is given by the quantum aspect of the Joule-Lenz law [6]-[8] the essence of which is expressed by the formula

$$
\Delta E_{n} \Delta t_{n}=h
$$

here $h$ is the Planck constant.

Because of (13) the emission intensity for transitions between the neighbouring quantum levels is

$$
I_{n+1, n}=\frac{\Delta E_{n}}{\Delta t_{n}}=\frac{\left(\Delta E_{n}\right)^{2}}{h} .
$$

If the energy distance between the levels corresponds to the next-neighbouring states we have

$$
I_{n+2, n}=\frac{E_{n+2}-E_{n}}{t_{n+2}-t_{n}}=\frac{\Delta E_{n}+\Delta E_{n+1}}{\Delta t_{n}+\Delta t_{n+1}} .
$$

Because of the relations (13) as well as

$$
E_{n+2}-E_{n}=E_{n+2}-E_{n+1}+E_{n+1}-E_{n}=\Delta E_{n+1}+\Delta E_{n}
$$

and

$$
t_{n+2}-t_{n}=t_{n+2}-t_{n+1}+t_{n+1}-t_{n}=\Delta t_{n+1}+\Delta t_{n},
$$


the formula (15) can be transformed into

$$
I_{n+2, n}=\frac{\Delta E_{n}+\Delta E_{n+1}}{h\left(\frac{1}{\Delta E_{n}}+\frac{1}{\Delta E_{n+1}}\right)}=\frac{\Delta E_{n} \Delta E_{n+1}}{h} .
$$

For the case of more distant quantum levels than $n+2$ and $n$, for example $n+q$ and $n$ where $q>2$, we have

$$
I_{n+q, n}=\frac{\Delta E_{n}+\Delta E_{n+1}+\cdots+\Delta E_{n+q-1}}{\Delta t_{n}+\Delta t_{n+1}+\cdots+\Delta t_{n+q-1}}=\frac{\Delta E_{n}+\Delta E_{n+1}+\cdots+\Delta E_{n+q-1}}{h\left(\frac{1}{\Delta E_{n}}+\frac{1}{\Delta E_{n+1}}+\cdots+\frac{1}{\Delta E_{n+q-1}}\right)} .
$$

Particular ratios of the emission intensity belonging to different pairs of the electron transitions in the hydrogen atom are represented in Table 1 and Table 3; see also [8]. In Table 1 are given the ratios

$$
\frac{I_{n_{\alpha}^{\prime} d}-I_{n_{\alpha}^{\prime \prime} P}}{I_{n_{\beta}^{\prime} d}-I_{n_{\beta}^{\prime \prime} p}}, \quad \frac{I_{n_{\alpha}^{\prime} f}-I_{n_{\alpha}^{\prime \prime} d}}{I_{n_{\beta}^{\prime} f}-I_{n_{\beta}^{\prime \prime} d}}, \quad \frac{I_{n_{\alpha}^{\prime} g}-I_{n_{\alpha}^{\prime \prime} f}}{I_{n_{\beta}^{\prime} g}-I_{n_{\beta}^{\prime \prime} f}} .
$$

Certainly $f$ in (20) should not be confused with $f$ in (5).

A characteristic point in (20) is that the angular momentum of the beginning state $n^{\prime}$ is larger than the angular momentum of the end state $n^{\prime \prime}$. In Table 3 are represented the intensity ratios

$$
\frac{I_{n_{\alpha}^{\prime} s}-I_{n_{\alpha}^{\prime \prime} p}}{I_{n_{\beta}^{\prime} s}-I_{n_{\beta}^{\prime \prime} p}},
$$

which correspond to transitions between the higher energy states having the angular momentum $s \quad(l=0)$ and lower energy states $p \quad(l=1)$. This is a case representing the angular momentum behaviour opposite to that given in (20).

\section{Quantum-Mechanical Ratios of Transition Probabilities between the Quantum States Examined in Table 1 and Table 3}

A quantum-mechanical counterpart of the semiclassical ratios of intensity considered in Sec. 2 and in Table 1 and Table 3 are the ratios of the transition probabilities calculated for the same sets of the quantum levels in [5]. These ratios are presented in Table 2 and Table 4. In Table 2 the intensity ratios of Table 1 are compared with the quantum-mechanical ratios of the transition probabilities which are

$$
\frac{A\left(n_{\alpha}^{\prime} d, n_{\alpha}^{\prime \prime} p\right)}{A\left(n_{\beta}^{\prime} d, n_{\beta}^{\prime \prime} p\right)}, \quad \frac{A\left(n_{\alpha}^{\prime} f, n_{\alpha}^{\prime \prime} d\right)}{A\left(n_{\beta}^{\prime} f, n_{\beta}^{\prime \prime} d\right)}, \quad \frac{A\left(n_{\alpha}^{\prime} g, n_{\alpha}^{\prime \prime} f\right)}{A\left(n_{\beta}^{\prime} g, n_{\beta}^{\prime \prime} f\right)} .
$$

On the other hand the semiclassical intensity ratios of Table 3 are compared with the quantum-mechanical ratios of transition probabilities [5]

$$
\frac{A\left(n_{\alpha}^{\prime} s, n_{\alpha}^{\prime \prime} p\right)}{A\left(n_{\beta}^{\prime} s, n_{\beta}^{\prime \prime} p\right)}
$$

in Table 4.

\section{Discussion of Results Presented in Tables 1-4}

In general the semiclassical intensity ratios presented in Table 1 differ solely by few percent from the quantum-mechanical ratios calculated in Table 2. The largest discrepancy between the semiclassical ratios of Table 1 and quantum-mechanical ratios of Table 2 seems to be in the case (61) where (see Table 2):

$$
\frac{\text { quantum-mechanical ratio }}{\text { semiclassical ratio }}=\frac{3.10}{1.84}=1.68>1.5 \text {. }
$$


Table 1. Intensity ratios of electron transitions between the $d$ and $p$ states, $f$ and $d$ states, and $g$ and $f$ states of the hydrogen atom calculated in a semiclassical way. The applied intervals of energy are presented in (4)-(6) and (9)-(11). The results are compared with the ratios of quantum-mechanical transition probabilities in Table 2.

\begin{tabular}{|c|c|c|c|}
\hline No. & Case & & Formula for the intensity ratio and the value of that ratio \\
\hline$(1)$ & $\frac{3 d-2 p}{4 d-2 p}$ & $\rightarrow$ & $\frac{\left(\Delta E_{2}\right)^{2}}{\Delta E_{2} \Delta E_{3}}=\frac{\Delta E_{2}}{\Delta E_{3}}=2.86$ \\
\hline$(2)$ & $\frac{3 d-2 p}{4 d-3 p}$ & $\rightarrow$ & $\frac{\left(\Delta E_{2}\right)^{2}}{\left(\Delta E_{3}\right)^{2}}=8.16$ \\
\hline (3) & $\frac{3 d-2 p}{5 d-2 p}$ & $\rightarrow$ & $\frac{\left(\Delta E_{2}\right)^{2}}{\Delta E_{5-2}}\left(\frac{1}{\Delta E_{2}}+\frac{1}{\Delta E_{3}}+\frac{1}{\Delta E_{4}}\right)=6.63$, \\
\hline$(4)$ & $\frac{3 d-2 p}{5 d-3 p}$ & $\rightarrow$ & $\frac{\left(\Delta E_{2}\right)^{2}}{\Delta E_{3} \Delta E_{4}}=17.6$ \\
\hline (5) & $\frac{3 d-2 p}{5 d-4 p}$ & $\rightarrow$ & $\frac{\left(\Delta E_{2}\right)^{2}}{\left(\Delta E_{4}\right)^{2}}=38.1$ \\
\hline (6) & $\frac{3 d-2 p}{6 d-2 p}$ & $\rightarrow$ & $\frac{\left(\Delta E_{2}\right)^{2}}{\Delta E_{6-2}}\left(\frac{1}{\Delta E_{2}}+\frac{1}{\Delta E_{3}}+\frac{1}{\Delta E_{4}}+\frac{1}{\Delta E_{5}}\right)=13.4$ \\
\hline (7) & $\frac{3 d-2 p}{6 d-3 p}$ & $\rightarrow$ & $\frac{\left(\Delta E_{2}\right)^{2}}{\Delta E_{6-3}}\left(\frac{1}{\Delta E_{3}}+\frac{1}{\Delta E_{4}}+\frac{1}{\Delta E_{5}}\right)=34.0$ \\
\hline$(8)$ & $\frac{3 d-2 p}{6 d-4 p}$ & $\rightarrow$ & $\frac{\left(\Delta E_{2}\right)^{2}}{\Delta E_{4} \Delta E_{5}}=70.1$ \\
\hline (9) & $\frac{3 d-2 p}{6 d-5 p}$ & $\rightarrow$ & $\frac{\left(\Delta E_{2}\right)^{2}}{\left(\Delta E_{5}\right)^{2}}=129$ \\
\hline$(10)$ & $\frac{4 d-2 p}{4 d-3 p}$ & $\rightarrow$ & $\frac{\Delta E_{2} \Delta E_{3}}{\left(\Delta E_{3}\right)^{2}}=\frac{\Delta E_{2}}{\Delta E_{3}}=2.86$ \\
\hline (11) & $\frac{4 d-2 p}{5 d-2 p}$ & $\rightarrow$ & $\frac{\Delta E_{2} \Delta E_{3}}{\Delta E_{5-2}}\left(\frac{1}{\Delta E_{2}}+\frac{1}{\Delta E_{3}}+\frac{1}{\Delta E_{4}}\right)=2.32$ \\
\hline$(12)$ & $\frac{4 d-2 p}{5 d-3 p}$ & $\rightarrow$ & $\frac{\Delta E_{2} \Delta E_{3}}{\Delta E_{3} \Delta E_{4}}=\frac{\Delta E_{2}}{\Delta E_{4}}=6.17$ \\
\hline (13) & $\frac{4 d-2 p}{5 d-4 p}$ & $\rightarrow$ & $\frac{\Delta E_{2} \Delta E_{3}}{\left(\Delta E_{4}\right)^{2}}=13.3$ \\
\hline$(14)$ & $\frac{4 d-2 p}{6 d-2 p}$ & $\rightarrow$ & $\frac{\Delta E_{2} \Delta E_{3}}{\Delta E_{6-2}}\left(\frac{1}{\Delta E_{2}}+\frac{1}{\Delta E_{3}}+\frac{1}{\Delta E_{4}}+\frac{1}{\Delta E_{5}}\right)=4.68$ \\
\hline$(15)$ & $\frac{4 d-2 p}{6 d-3 p}$ & $\rightarrow$ & $\frac{\Delta E_{2} \Delta E_{3}}{\Delta E_{6-3}}\left(\frac{1}{\Delta E_{3}}+\frac{1}{\Delta E_{4}}+\frac{1}{\Delta E_{5}}\right)=11.9$ \\
\hline (16) & $\frac{4 d-2 p}{6 d-4 p}$ & $\rightarrow$ & $\frac{\Delta E_{2} \Delta E_{3}}{\Delta E_{4} \Delta E_{5}}=24.6$ \\
\hline (17) & $\frac{4 d-2 p}{6 d-5 p}$ & $\rightarrow$ & $\frac{\Delta E_{2} \Delta E_{3}}{\left(\Delta E_{5}\right)^{2}}=45.2$ \\
\hline (18) & $\frac{4 d-3 p}{5 d-2 p}$ & $\rightarrow$ & $\frac{\left(\Delta E_{3}\right)^{2}}{\Delta E_{5-2}}\left(\frac{1}{\Delta E_{2}}+\frac{1}{\Delta E_{3}}+\frac{1}{\Delta E_{4}}\right)=0.81$, \\
\hline (19) & $\frac{4 d-3 p}{5 d-3 p}$ & $\rightarrow$ & $\frac{\left(\Delta E_{3}\right)^{2}}{\Delta E_{3} \Delta E_{4}}=\frac{\Delta E_{3}}{\Delta E_{4}}=2.16$ \\
\hline$(20)$ & $\frac{4 d-3 p}{5 d-4 p}$ & $\rightarrow$ & $\frac{\left(\Delta E_{3}\right)^{2}}{\left(\Delta E_{4}\right)^{2}}=4.67$ \\
\hline
\end{tabular}




\section{Continued}

(21) $\frac{4 d-3 p}{6 d-2 p} \rightarrow \frac{\left(\Delta E_{3}\right)^{2}}{\Delta E_{6-2}}\left(\frac{1}{\Delta E_{2}}+\frac{1}{\Delta E_{3}}+\frac{1}{\Delta E_{4}}+\frac{1}{\Delta E_{5}}\right)=1.64$,

(22) $\frac{4 d-3 p}{6 d-3 p} \rightarrow \frac{\left(\Delta E_{3}\right)^{2}}{\Delta E_{6-3}}\left(\frac{1}{\Delta E_{3}}+\frac{1}{\Delta E_{4}}+\frac{1}{\Delta E_{5}}\right)=4.16$,

(23) $\frac{4 d-3 p}{6 d-4 p} \rightarrow \frac{\left(\Delta E_{3}\right)^{2}}{\Delta E_{4} \Delta E_{5}}=8.59$,

(24) $\quad \frac{4 d-3 p}{6 d-5 p} \rightarrow \frac{\left(\Delta E_{3}\right)^{2}}{\left(\Delta E_{5}\right)^{2}}=15.8$,

(25) $\frac{5 d-2 p}{5 d-3 p} \rightarrow \frac{\Delta E_{5-2}}{\frac{1}{\Delta E_{2}}+\frac{1}{\Delta E_{3}}+\frac{1}{\Delta E_{4}}} \frac{1}{\Delta E_{3} \Delta E_{4}}=2.66$,

$\frac{5 d-2 p}{5 d-4 p} \rightarrow \frac{\Delta E_{5-2}}{\frac{1}{\Delta E_{2}}+\frac{1}{\Delta E_{3}}+\frac{1}{\Delta E_{4}}} \frac{1}{\left(\Delta E_{4}\right)^{2}}=5.74$,$$
\frac{5 d-2 p}{6 d-2 p} \rightarrow \frac{\Delta E_{5-2}}{\frac{1}{\Delta E_{2}}+\frac{1}{\Delta E_{3}}+\frac{1}{\Delta E_{4}}} \frac{1}{\Delta E_{6-2}}\left(\frac{1}{\Delta E_{2}}+\frac{1}{\Delta E_{3}}+\frac{1}{\Delta E_{4}}+\frac{1}{\Delta E_{5}}\right)=2.02,
$$$$
\frac{5 d-2 p}{6 d-3 p} \rightarrow \frac{\Delta E_{5-2}}{\frac{1}{\Delta E_{2}}+\frac{1}{\Delta E_{3}}+\frac{1}{\Delta E_{4}}} \frac{1}{\Delta E_{6-3}}\left(\frac{1}{\Delta E_{3}}+\frac{1}{\Delta E_{4}}+\frac{1}{\Delta E_{5}}\right)=5.12,
$$$$
\frac{5 d-2 p}{6 d-4 p} \rightarrow \frac{\Delta E_{5-2}}{\frac{1}{\Delta E_{2}}+\frac{1}{\Delta E_{3}}+\frac{1}{\Delta E_{4}}} \frac{1}{\Delta E_{4} \Delta E_{5}}=10.6,
$$$$
\frac{5 d-2 p}{6 d-5 p} \rightarrow \frac{\Delta E_{5-2}}{\frac{1}{\Delta E_{2}}+\frac{1}{\Delta E_{3}}+\frac{1}{\Delta E_{4}}} \frac{1}{\left(\Delta E_{5}\right)^{2}}=19.5,
$$$$
\frac{5 d-3 p}{5 d-4 p} \quad \rightarrow \quad \frac{\Delta E_{3} \Delta E_{4}}{\left(\Delta E_{4}\right)^{2}}=\frac{\Delta E_{3}}{\Delta E_{4}}=2.16
$$$$
\frac{5 d-3 p}{6 d-2 p} \rightarrow \frac{\Delta E_{3} \Delta E_{4}}{\Delta E_{6-2}}\left(\frac{1}{\Delta E_{2}}+\frac{1}{\Delta E_{3}}+\frac{1}{\Delta E_{4}}+\frac{1}{\Delta E_{5}}\right)=0.76,
$$$$
\frac{5 d-3 p}{6 d-3 p} \rightarrow \frac{\Delta E_{3} \Delta E_{4}}{\Delta E_{6-3}}\left(\frac{1}{\Delta E_{3}}+\frac{1}{\Delta E_{4}}+\frac{1}{\Delta E_{5}}\right)=1.93 \text {, }
$$$$
\text { 34) } \frac{5 d-3 p}{6 d-4 p} \rightarrow \frac{\Delta E_{3} \Delta E_{4}}{\Delta E_{4} \Delta E_{5}}=\frac{\Delta E_{3}}{\Delta E_{5}}=3.98 \text {, }
$$$$
\text { 5) } \frac{5 d-3 p}{6 d-5 p} \rightarrow \frac{\Delta E_{3} \Delta E_{4}}{\left(\Delta E_{5}\right)^{2}}=7.32 \text {, }
$$$$
\text { 6) } \frac{5 d-4 p}{6 d-2 p} \rightarrow \frac{\left(\Delta E_{4}\right)^{2}}{\Delta E_{6-2}}\left(\frac{1}{\Delta E_{2}}+\frac{1}{\Delta E_{3}}+\frac{1}{\Delta E_{4}}+\frac{1}{\Delta E_{5}}\right)=0.35 \text {, }
$$$$
\text { 37) } \frac{5 d-4 p}{6 d-3 p} \rightarrow \frac{\left(\Delta E_{4}\right)^{2}}{\Delta E_{6-3}}\left(\frac{1}{\Delta E_{3}}+\frac{1}{\Delta E_{4}}+\frac{1}{\Delta E_{5}}\right)=0.89 \text {, }
$$$$
\text { (38) } \frac{5 d-4 p}{6 d-4 p} \rightarrow \frac{\left(\Delta E_{4}\right)^{2}}{\Delta E_{4} \Delta E_{5}}=\frac{\Delta E_{4}}{\Delta E_{5}}=1.84 \text {, }
$$

$$
\frac{5 d-4 p}{6 d-5 p} \rightarrow \frac{\left(\Delta E_{4}\right)^{2}}{\left(\Delta E_{5}\right)^{2}}=3.39
$$




\section{Continued}

(40)

$$
\frac{6 d-2 p}{6 d-3 p} \rightarrow \frac{\Delta E_{6-2}}{\frac{1}{\Delta E_{2}}+\frac{1}{\Delta E_{3}}+\frac{1}{\Delta E_{4}}+\frac{1}{\Delta E_{5}}} \frac{1}{\Delta E_{6-3}}\left(\frac{1}{\Delta E_{3}}+\frac{1}{\Delta E_{4}}+\frac{1}{\Delta E_{5}}\right)=2.54
$$

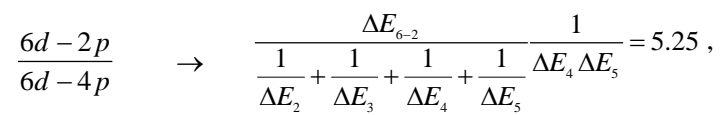

(42)

$\frac{6 d-2 p}{6 d-5 p} \rightarrow \frac{\Delta E_{6-2}}{\frac{1}{\Delta E_{2}}+\frac{1}{\Delta E_{3}}+\frac{1}{\Delta E_{4}}+\frac{1}{\Delta E_{5}}} \frac{1}{\left(\Delta E_{5}\right)^{2}}=9.66$,

$$
\frac{6 d-3 p}{6 d-4 p} \rightarrow \frac{\Delta E_{6-3}}{\frac{1}{\Delta E_{3}}+\frac{1}{\Delta E_{4}}+\frac{1}{\Delta E_{5}}} \frac{1}{\Delta E_{4} \Delta E_{5}}=2.06
$$

$$
\frac{6 d-3 p}{6 d-5 p} \rightarrow \frac{\Delta E_{6-3}}{\frac{1}{\Delta E_{3}}+\frac{1}{\Delta E_{4}}+\frac{1}{\Delta E_{5}}} \frac{1}{\left(\Delta E_{5}\right)^{2}}=3.80,
$$

$$
\frac{6 d-4 p}{6 d-5 p} \quad \rightarrow \quad \frac{\Delta E_{4} \Delta E_{5}}{\left(\Delta E_{5}\right)^{2}}=\frac{\Delta E_{4}}{\Delta E_{5}}=1.84
$$

$$
\frac{4 f-3 d}{5 f-3 d} \rightarrow \frac{\left(\Delta E_{3}\right)^{2}}{\Delta E_{3} \Delta E_{4}}=\frac{\Delta E_{3}}{\Delta E_{4}}=2.16
$$

$$
\frac{4 f-3 d}{5 f-4 d} \rightarrow \frac{\left(\Delta E_{3}\right)^{2}}{\left(\Delta E_{4}\right)^{2}}=4.67,
$$

$$
\frac{4 f-3 d}{6 f-3 d} \rightarrow \frac{\left(\Delta E_{3}\right)^{2}}{\Delta E_{6-3}}\left(\frac{1}{\Delta E_{3}}+\frac{1}{\Delta E_{4}}+\frac{1}{\Delta E_{5}}\right)=4.16,
$$

$$
\frac{4 f-3 d}{6 f-4 d} \rightarrow \frac{\left(\Delta E_{3}\right)^{2}}{\Delta E_{4} \Delta E_{5}}=8.59,
$$

$\frac{4 f-3 d}{6 f-5 d} \rightarrow \frac{\left(\Delta E_{3}\right)^{2}}{\left(\Delta E_{5}\right)^{2}}=15.8$,

1) $\frac{5 f-3 d}{5 f-4 d} \rightarrow \frac{\Delta E_{3} \Delta E_{4}}{\left(\Delta E_{4}\right)^{2}}=\frac{\Delta E_{3}}{\Delta E_{4}}=2.16$,

$\frac{5 f-3 d}{6 f-3 d} \rightarrow \frac{\Delta E_{3} \Delta E_{4}}{\Delta E_{6-3}}\left(\frac{1}{\Delta E_{3}}+\frac{1}{\Delta E_{4}}+\frac{1}{\Delta E_{5}}\right)=1.93$,

$\frac{5 f-3 d}{6 f-4 d} \rightarrow \frac{\Delta E_{3} \Delta E_{4}}{\Delta E_{4} \Delta E_{5}}=\frac{\Delta E_{3}}{\Delta E_{5}}=3.98$,

$\frac{5 f-3 d}{6 f-5 d} \rightarrow \frac{\Delta E_{3} \Delta E_{4}}{\left(\Delta E_{5}\right)^{2}}=7.32$,

$$
\frac{5 f-4 d}{6 f-3 d} \quad \rightarrow \quad \frac{\left(\Delta E_{4}\right)^{2}}{\Delta E_{6-3}}\left(\frac{1}{\Delta E_{3}}+\frac{1}{\Delta E_{4}}+\frac{1}{\Delta E_{5}}\right)=0.89,
$$

$\frac{5 f-4 d}{6 f-4 d} \rightarrow \frac{\left(\Delta E_{4}\right)^{2}}{\Delta E_{4} \Delta E_{5}}=\frac{\Delta E_{4}}{\Delta E_{5}}=1.84$,

$\frac{5 f-4 d}{6 f-5 d} \rightarrow \frac{\left(\Delta E_{4}\right)^{2}}{\left(\Delta E_{5}\right)^{2}}=3.39$

$$
\frac{6 f-3 d}{6 f-4 d} \rightarrow \frac{\Delta E_{6-3}}{\frac{1}{\Delta E_{3}}+\frac{1}{\Delta E_{4}}+\frac{1}{\Delta E_{5}}} \frac{1}{\Delta E_{4} \Delta E_{5}}=2.06,
$$




\section{Continued}
(59)
$\frac{6 f-3 d}{6 f-5 d} \rightarrow \frac{\Delta E_{6-3}}{\frac{1}{\Delta E_{3}}+\frac{1}{\Delta E_{4}}+\frac{1}{\Delta E_{5}}} \frac{1}{\left(\Delta E_{5}\right)^{2}}=3.80$,
(60)
$\frac{6 f-4 d}{6 f-5 d} \rightarrow \frac{\Delta E_{4} \Delta E_{5}}{\left(\Delta E_{5}\right)^{2}}=\frac{\Delta E_{4}}{\Delta E_{5}}=1.84$,
(61)
$\frac{5 g-4 f}{6 g-4 f} \rightarrow \frac{\left(\Delta E_{4}\right)^{2}}{\Delta E_{4} \Delta E_{5}}=\frac{\Delta E_{4}}{\Delta E_{5}}=1.84$,
(62) $\frac{5 g-4 f}{6 g-5 f} \rightarrow \frac{\left(\Delta E_{4}\right)^{2}}{\left(\Delta E_{5}\right)^{2}}=3.39$,
(63)
$\frac{6 g-4 f}{6 g-5 f} \rightarrow \frac{\Delta E_{4} \Delta E_{5}}{\left(\Delta E_{5}\right)^{2}}=1.84$.

Table 2. Quantum-mechanical ratios of the transition probabilities between the pairs of quantum states examined in Table 1 (see [5]) compared with the intensity ratios calculated in Table 1.

\begin{tabular}{|c|c|c|c|c|}
\hline No. & Case & & Quantum-mechanical ratio & Intensity ratio from Table 1 \\
\hline (1) & $\frac{3 d-2 p}{4 d-2 p}$ & $\rightarrow$ & $\frac{0.64}{0.204}=3.14$ & 2.86 \\
\hline$(2)$ & $\frac{3 d-2 p}{4 d-3 p}$ & $\rightarrow$ & $\frac{0.64}{0.07}=9.14$; & 8.16 \\
\hline (3) & $\frac{3 d-2 p}{5 d-2 p}$ & $\rightarrow$ & $\frac{0.64}{0.094}=6.81$ & 6.63 \\
\hline (4) & $\frac{3 d-2 p}{5 d-3 p}$ & $\rightarrow$ & $\frac{0.64}{0.034}=18.8$ & 17.6 \\
\hline (5) & $\frac{3 d-2 p}{5 d-4 p}$ & $\rightarrow$ & $\frac{0.64}{0.014}=45.7$ & 38.1 \\
\hline (6) & $\frac{3 d-2 p}{6 d-2 p}$ & $\rightarrow$ & $\frac{0.64}{0.048}=13.3$ & 13.4 \\
\hline (7) & $\frac{3 d-2 p}{6 d-3 p}$ & $\rightarrow$ & $\frac{0.64}{0.0187}=34.2$ & 34.0 \\
\hline (8) & $\frac{3 d-2 p}{6 d-4 p}$ & $\rightarrow$ & $\frac{0.64}{0.0086}=74.4$; & 70.1 \\
\hline (9) & $\frac{3 d-2 p}{6 d-5 p}$ & $\rightarrow$ & $\frac{0.64}{0.0040}=160$ & 129 \\
\hline (10) & $\frac{4 d-2 p}{4 d-3 p}$ & $\rightarrow$ & $\frac{0.204}{0.070}=2.91$ & 2.86 \\
\hline (11) & $\frac{4 d-2 p}{5 d-2 p}$ & $\rightarrow$ & $\frac{0.204}{0.094}=2.17$ & 2.32 \\
\hline (12) & $\frac{4 d-2 p}{5 d-3 p}$ & $\rightarrow$ & $\frac{0.204}{0.034}=6.00$; & 6.17 \\
\hline (13) & $\frac{4 d-2 p}{5 d-4 p}$ & $\rightarrow$ & $\frac{0.204}{0.014}=14.6$ & 13.3 \\
\hline (14) & $\frac{4 d-2 p}{6 d-2 p}$ & $\rightarrow$ & $\frac{0.204}{0.048}=4.25$ & 4.68 \\
\hline (15) & $\frac{4 d-2 p}{6 d-3 p}$ & $\rightarrow$ & $\frac{0.204}{0.0187}=10.9$ & 11.9 \\
\hline (16) & $\frac{4 d-2 p}{6 d-4 p}$ & $\rightarrow$ & $\frac{0.204}{0.0086}=23.7$; & 24.6 \\
\hline
\end{tabular}




\section{Continued}

(17)

$$
\frac{4 d-2 p}{6 d-5 p} \rightarrow \frac{0.204}{0.0040}=51 ;
$$

$$
\frac{4 d-3 p}{5 d-2 p} \quad \rightarrow \quad \frac{0.070}{0.094}=0.74 ;
$$

$$
\frac{4 d-3 p}{5 d-3 p} \quad \rightarrow \quad \frac{0.070}{0.034}=2.06 ;
$$

(20)

$$
\frac{4 d-3 p}{5 d-4 p} \rightarrow \quad \frac{0.070}{0.014}=5.0 ;
$$

$$
\frac{4 d-3 p}{6 d-2 p} \rightarrow \frac{0.070}{0.048}=1.46 ;
$$

$$
\frac{4 d-3 p}{6 d-3 p} \rightarrow \frac{0.070}{0.0187}=3.74 ;
$$

$$
\frac{4 d-3 p}{6 d-4 p} \rightarrow \frac{0.070}{0.0086}=8.14 ;
$$

$$
\frac{4 d-3 p}{6 d-5 p} \rightarrow \frac{0.070}{0.0040}=17.5 ;
$$

$$
\frac{5 d-2 p}{5 d-3 p} \quad \rightarrow \quad \frac{0.094}{0.034}=2.76
$$

$$
\frac{5 d-2 p}{5 d-4 p} \quad \rightarrow \quad \frac{0.094}{0.014}=6.71
$$

$$
\frac{5 d-2 p}{6 d-2 p} \quad \rightarrow \quad \frac{0.094}{0.048}=1.96
$$

$$
\frac{5 d-2 p}{6 d-3 p} \rightarrow \frac{0.094}{0.0187}=5.03 ;
$$

$$
\frac{5 d-2 p}{6 d-4 p} \rightarrow \frac{0.094}{0.0086}=10.9 ;
$$

$$
\frac{5 d-2 p}{6 d-5 p} \quad \rightarrow \quad \frac{0.094}{0.0040}=23.5 \text {; }
$$

$$
\frac{5 d-3 p}{5 d-4 p} \quad \rightarrow \quad \frac{0.034}{0.014}=2.43 ;
$$

$$
\frac{5 d-3 p}{6 d-2 p} \quad \rightarrow \quad \frac{0.034}{0.048}=0.71
$$

$$
\frac{5 d-3 p}{6 d-3 p} \quad \rightarrow \quad \frac{0.034}{0.0187}=1.82 ;
$$

$$
\frac{5 d-3 p}{6 d-4 p} \rightarrow \frac{0.034}{0.0086}=3.95 ;
$$

$$
\frac{5 d-3 p}{6 d-5 p} \quad \rightarrow \quad \frac{0.034}{0.0040}=8.5 ;
$$

$$
\frac{5 d-4 p}{6 d-2 p} \quad \rightarrow \quad \frac{0.014}{0.048}=0.29 ;
$$

$$
\frac{5 d-4 p}{6 d-3 p} \rightarrow \frac{0.014}{0.0187}=0.75 ;
$$

$$
\frac{5 d-4 p}{6 d-4 p} \quad \rightarrow \quad \frac{0.014}{0.0086}=1.63 ;
$$

$$
\frac{5 d-4 p}{6 d-5 p} \quad \rightarrow \quad \frac{0.014}{0.0040}=3.5 ;
$$




\section{Continued}

(41)

$$
\frac{6 d-2 p}{6 d-4 p} \quad \rightarrow \quad \frac{0.048}{0.0086}=5.58 ;
$$

(42)

$$
\frac{6 d-2 p}{6 d-5 p} \quad \rightarrow \quad \frac{0.048}{0.0040}=12.0 ;
$$

(43)

$$
\frac{6 d-3 p}{6 d-4 p} \quad \rightarrow \quad \frac{0.0187}{0.0086}=2.17
$$

$$
\frac{6 d-3 p}{6 d-5 p} \quad \rightarrow \quad \frac{0.0187}{0.0040}=4.68
$$

$$
\frac{6 d-4 p}{6 d-5 p} \quad \rightarrow \quad \frac{0.0086}{0.0040}=2.15 ;
$$

$$
\frac{4 f-3 d}{5 f-3 d} \quad \rightarrow \quad \frac{0.137}{0.045}=3.04
$$

$$
\frac{4 f-3 d}{5 f-4 d} \quad \rightarrow \quad \frac{0.137}{0.026}=5.27
$$

(48)

$$
\frac{4 f-3 d}{6 f-3 d} \quad \rightarrow \quad \frac{0.137}{0.021}=6.52
$$

$$
\frac{5 f-3 d}{5 f-4 d} \quad \rightarrow \quad \frac{0.045}{0.026}=1.73
$$

$$
\frac{5 f-3 d}{6 f-3 d} \quad \rightarrow \quad \frac{0.045}{0.021}=2.14 ;
$$

$$
\frac{5 f-3 d}{6 f-4 d} \quad \rightarrow \quad \frac{0.045}{0.0129}=3.49 ;
$$

$$
\frac{5 f-3 d}{6 f-5 d} \quad \rightarrow \quad \frac{0.045}{0.0072}=6.25 \text {; }
$$$$
\frac{5 f-4 d}{6 f-3 d} \quad \rightarrow \quad \frac{0.026}{0.021}=1.24 \text {; }
$$

$$
\frac{5 g-4 f}{6 g-4 f} \rightarrow \frac{0.0425}{0.0137}=3.10 ;
$$


Table 3. Intensity ratios of the electron transitions between the $s$ and $p$ states of the atomic hydrogen calculated in a semiclassical way; states $s$ have here higher energy than states $p$. A comparison of the results of the present Table with the ratios of quantum-mechanical transition probabilities is done in Table 4.

\begin{tabular}{|c|c|c|c|}
\hline No. & Case & & Formula for the intensity ratio and the value of that ratio \\
\hline$(1)$ & $\frac{3 s-2 p}{4 s-2 p}$ & $\rightarrow$ & $\frac{\left(\Delta E_{2}\right)^{2}}{\Delta E_{2} \Delta E_{3}}=\frac{\Delta E_{2}}{\Delta E_{3}}=2.86$ \\
\hline$(2)$ & $\frac{3 s-2 p}{4 s-3 p}$ & $\rightarrow$ & $\frac{\left(\Delta E_{2}\right)^{2}}{\left(\Delta E_{3}\right)^{2}}=8.16$ \\
\hline (3) & $\frac{3 s-2 p}{5 s-2 p}$ & $\rightarrow$ & $\frac{\left(\Delta E_{2}\right)^{2}}{\Delta E_{5-2}}\left(\frac{1}{\Delta E_{2}}+\frac{1}{\Delta E_{3}}+\frac{1}{\Delta E_{4}}\right)=6.63$, \\
\hline (4) & $\frac{3 s-2 p}{5 s-3 p}$ & $\rightarrow$ & $\frac{\left(\Delta E_{2}\right)^{2}}{\Delta E_{3} \Delta E_{4}}=17.6$ \\
\hline$(5)$ & $\frac{3 s-2 p}{5 s-4 p}$ & $\rightarrow$ & $\frac{\left(\Delta E_{2}\right)^{2}}{\left(\Delta E_{4}\right)^{2}}=38.1$ \\
\hline (6) & $\frac{3 s-2 p}{6 s-2 p}$ & $\rightarrow$ & $\frac{\left(\Delta E_{2}\right)^{2}}{\Delta E_{6-2}}\left(\frac{1}{\Delta E_{2}}+\frac{1}{\Delta E_{3}}+\frac{1}{\Delta E_{4}}+\frac{1}{\Delta E_{5}}\right)=13.4$, \\
\hline$(7)$ & $\frac{3 s-2 p}{6 s-3 p}$ & $\rightarrow$ & $\frac{\left(\Delta E_{2}\right)^{2}}{\Delta E_{6-3}}\left(\frac{1}{\Delta E_{3}}+\frac{1}{\Delta E_{4}}+\frac{1}{\Delta E_{5}}\right)=4.16$, \\
\hline (8) & $\frac{3 s-2 p}{6 s-4 p}$ & $\rightarrow$ & $\frac{\left(\Delta E_{2}\right)^{2}}{\Delta E_{4} \Delta E_{5}}=70.1$ \\
\hline (9) & $\frac{3 s-2 p}{6 s-5 p}$ & $\rightarrow$ & $\frac{\left(\Delta E_{2}\right)^{2}}{\left(\Delta E_{5}\right)^{2}}=129$ \\
\hline$(10)$ & $\frac{4 s-2 p}{4 s-3 p}$ & $\rightarrow$ & $\frac{\Delta E_{2} \Delta E_{3}}{\left(\Delta E_{3}\right)^{2}}=\frac{\Delta E_{2}}{\Delta E_{3}}=2.86$ \\
\hline$(11)$ & $\frac{4 s-2 p}{5 s-2 p}$ & $\rightarrow$ & $\frac{\Delta E_{2} \Delta E_{3}}{\Delta E_{5-2}}\left(\frac{1}{\Delta E_{2}}+\frac{1}{\Delta E_{3}}+\frac{1}{\Delta E_{4}}\right)=2.32$, \\
\hline$(12)$ & $\frac{4 s-2 p}{5 s-3 p}$ & $\rightarrow$ & $\frac{\Delta E_{2} \Delta E_{3}}{\Delta E_{3} \Delta E_{4}}=\frac{\Delta E_{2}}{\Delta E_{4}}=6.17$ \\
\hline (13) & $\frac{4 s-2 p}{5 s-4 p}$ & $\rightarrow$ & $\frac{\Delta E_{2} \Delta E_{3}}{\left(\Delta E_{4}\right)^{2}}=13.3$ \\
\hline$(14)$ & $\frac{4 s-2 p}{6 s-2 p}$ & $\rightarrow$ & $\frac{\Delta E_{2} \Delta E_{3}}{\Delta E_{6-2}}\left(\frac{1}{\Delta E_{2}}+\frac{1}{\Delta E_{3}}+\frac{1}{\Delta E_{4}}+\frac{1}{\Delta E_{5}}\right)=4.68$ \\
\hline$(15)$ & $\frac{4 s-3 p}{5 s-2 p}$ & $\rightarrow$ & $\frac{\left(\Delta E_{3}\right)^{2}}{\Delta E_{5-2}}\left(\frac{1}{\Delta E_{2}}+\frac{1}{\Delta E_{3}}+\frac{1}{\Delta E_{4}}\right)=0.81$ \\
\hline (16) & $\frac{4 s-3 p}{5 s-3 p}$ & $\rightarrow$ & $\frac{\left(\Delta E_{3}\right)^{2}}{\Delta E_{3} \Delta E_{4}}=\frac{\Delta E_{3}}{\Delta E_{4}}=2.16$ \\
\hline$(17)$ & $\frac{4 s-3 p}{5 s-4 p}$ & $\rightarrow$ & $\frac{\left(\Delta E_{3}\right)^{2}}{\left(\Delta E_{4}\right)^{2}}=4.67$ \\
\hline (18) & $\frac{4 s-3 p}{6 s-2 p}$ & $\rightarrow$ & $\frac{\left(\Delta E_{3}\right)^{2}}{\Delta E_{6-2}}\left(\frac{1}{\Delta E_{2}}+\frac{1}{\Delta E_{3}}+\frac{1}{\Delta E_{4}}+\frac{1}{\Delta E_{5}}\right)=1.64$, \\
\hline (19) & $\frac{4 s-3 p}{6 s-3 p}$ & $\rightarrow$ & $\frac{\left(\Delta E_{3}\right)^{2}}{\Delta E_{6-3}}\left(\frac{1}{\Delta E_{3}}+\frac{1}{\Delta E_{4}}+\frac{1}{\Delta E_{5}}\right)=4.16$, \\
\hline$(20)$ & $\frac{4 s-3 p}{6 s-4 p}$ & $\rightarrow$ & $\frac{\left(\Delta E_{3}\right)^{2}}{\Delta E_{4} \Delta E_{5}}=8.59$ \\
\hline
\end{tabular}




\section{Continued}

(21)

$\frac{4 s-3 p}{6 s-5 p} \rightarrow \frac{\left(\Delta E_{3}\right)^{2}}{\left(\Delta E_{5}\right)^{2}}=15.8$

(22)

$\frac{5 s-2 p}{5 s-3 p} \rightarrow \frac{\Delta E_{5-2}}{\frac{1}{\Delta E_{2}}+\frac{1}{\Delta E_{3}}+\frac{1}{\Delta E_{4}}} \frac{1}{\Delta E_{3} \Delta E_{4}}=2.66$,

(23)

$\frac{5 s-2 p}{5 s-4 p} \rightarrow \frac{\Delta E_{5-2}}{\frac{1}{\Delta E_{2}}+\frac{1}{\Delta E_{3}}+\frac{1}{\Delta E_{4}}} \frac{1}{\left(\Delta E_{4}\right)^{2}}=5.74$,

(24)

$\frac{5 s-2 p}{6 s-2 p} \rightarrow \frac{\Delta E_{5-2}}{\frac{1}{\Delta E_{2}}+\frac{1}{\Delta E_{3}}+\frac{1}{\Delta E_{4}}}\left(\frac{1}{\Delta E_{2}}+\frac{1}{\Delta E_{3}}+\frac{1}{\Delta E_{4}}+\frac{1}{\Delta E_{5}}\right) \frac{1}{\Delta E_{6-2}}=2.02$,

(25)

$\frac{5 s-2 p}{6 s-3 p} \rightarrow \frac{\Delta E_{5-2}}{\frac{1}{\Delta E_{2}}+\frac{1}{\Delta E_{3}}+\frac{1}{\Delta E_{4}}}\left(\frac{1}{\Delta E_{3}}+\frac{1}{\Delta E_{4}}+\frac{1}{\Delta E_{5}}\right) \frac{1}{\Delta E_{6-3}}=5.12$,

(26)

$\frac{5 s-2 p}{6 s-4 p} \rightarrow \frac{\Delta E_{5-2}}{\frac{1}{\Delta E_{2}}+\frac{1}{\Delta E_{3}}+\frac{1}{\Delta E_{4}}} \frac{1}{\Delta E_{4} \Delta E_{5}}=10.58$,

(27)

$\frac{5 s-2 p}{6 s-5 p} \rightarrow \frac{\Delta E_{5-2}}{\frac{1}{\Delta E_{2}}+\frac{1}{\Delta E_{3}}+\frac{1}{\Delta E_{4}}} \frac{1}{\left(\Delta E_{5}\right)^{2}}=19.47$,

(28)

$\frac{5 s-3 p}{5 s-4 p} \rightarrow \frac{\Delta E_{3} \Delta E_{4}}{\left(\Delta E_{4}\right)^{2}}=\frac{\Delta E_{3}}{\Delta E_{4}}=2.16$,

(29)

$\frac{5 s-3 p}{6 s-2 p} \rightarrow \frac{\Delta E_{3} \Delta E_{4}}{\Delta E_{6-2}}\left(\frac{1}{\Delta E_{2}}+\frac{1}{\Delta E_{3}}+\frac{1}{\Delta E_{4}}+\frac{1}{\Delta E_{5}}\right)=0.76$,

(30)

$\frac{5 s-3 p}{6 s-3 p} \rightarrow \frac{\Delta E_{3} \Delta E_{4}}{\Delta E_{6-3}}\left(\frac{1}{\Delta E_{3}}+\frac{1}{\Delta E_{4}}+\frac{1}{\Delta E_{5}}\right)=1.93$,

(31)

$\frac{5 s-3 p}{6 s-4 p} \rightarrow \frac{\Delta E_{3} \Delta E_{4}}{\Delta E_{4} \Delta E_{5}}=\frac{\Delta E_{3}}{\Delta E_{5}}=3.98$,

$\frac{5 s-3 p}{6 s-5 p} \rightarrow \frac{\Delta E_{3} \Delta E_{4}}{\left(\Delta E_{5}\right)^{2}}=7.32$,

(33)

$\frac{5 s-4 p}{6 s-2 p} \rightarrow \frac{\left(\Delta E_{4}\right)^{2}}{\Delta E_{6-2}}\left(\frac{1}{\Delta E_{2}}+\frac{1}{\Delta E_{3}}+\frac{1}{\Delta E_{4}}+\frac{1}{\Delta E_{5}}\right)=0.35$,

(34)

$\frac{5 s-4 p}{6 s-3 p} \rightarrow \frac{\left(\Delta E_{4}\right)^{2}}{\Delta E_{6-3}}\left(\frac{1}{\Delta E_{3}}+\frac{1}{\Delta E_{4}}+\frac{1}{\Delta E_{5}}\right)=0.89$,

(35)

$\frac{5 s-4 p}{6 s-4 p} \rightarrow \frac{\left(\Delta E_{4}\right)^{2}}{\Delta E_{4} \Delta E_{5}}=\frac{\Delta E_{4}}{\Delta E_{5}}=1.84$,

(36)

$\frac{5 s-4 p}{6 s-5 p} \rightarrow \frac{\left(\Delta E_{4}\right)^{2}}{\left(\Delta E_{5}\right)^{2}}=3.39$,

(37)

$\frac{6 s-2 p}{6 s-3 p} \rightarrow \frac{\Delta E_{6-2}}{\frac{1}{\Delta E_{2}}+\frac{1}{\Delta E_{3}}+\frac{1}{\Delta E_{4}}+\frac{1}{\Delta E_{5}}}\left(\frac{1}{\Delta E_{3}}+\frac{1}{\Delta E_{4}}+\frac{1}{\Delta E_{5}}\right) \frac{1}{\Delta E_{6-3}}=2.54$,

(38)

$\frac{6 s-2 p}{6 s-4 p} \rightarrow \frac{\Delta E_{6-2}}{\frac{1}{\Delta E_{2}}+\frac{1}{\Delta E_{3}}+\frac{1}{\Delta E_{4}}+\frac{1}{\Delta E_{5}}} \frac{1}{\Delta E_{4} \Delta E_{5}}=5.25$

(39)

$$
\frac{6 s-2 p}{6 s-5 p} \rightarrow \frac{\Delta E_{6-2}}{\frac{1}{\Delta E_{2}}+\frac{1}{\Delta E_{3}}+\frac{1}{\Delta E_{4}}+\frac{1}{\Delta E_{5}}} \frac{1}{\left(\Delta E_{5}\right)^{2}}=9.66
$$




\section{Continued}

(40)

$$
\frac{6 s-3 p}{6 s-4 p} \rightarrow \frac{\Delta E_{6-3}}{\frac{1}{\Delta E_{3}}+\frac{1}{\Delta E_{4}}+\frac{1}{\Delta E_{5}}} \frac{1}{\Delta E_{4} \Delta E_{5}}=2.06
$$$$
\frac{6 s-3 p}{6 s-5 p} \rightarrow \frac{\Delta E_{6-3}}{\frac{1}{\Delta E_{3}}+\frac{1}{\Delta E_{4}}+\frac{1}{\Delta E_{5}}} \frac{1}{\left(\Delta E_{5}\right)^{2}}=3.80,
$$

$$
\frac{6 s-4 p}{6 s-5 p} \rightarrow \frac{\Delta E_{4} \Delta E_{5}}{\left(\Delta E_{5}\right)^{2}}=\frac{\Delta E_{4}}{\Delta E_{5}}=1.84
$$

Table 4. Quantum-mechanical ratios of the transition probabilities for the pairs of states examined in Table 3 compared with

\begin{tabular}{|c|c|c|c|c|}
\hline No. & Case & & Quantum-mechanical ratio & Intensity ratio from Table 3 \\
\hline (1) & $\frac{3 s-2 p}{4 s-2 p}$ & $\rightarrow$ & $\frac{0.063}{0.025}=2.52$ & 2.86 \\
\hline$(2)$ & $\frac{3 s-2 p}{4 s-3 p}$ & $\rightarrow$ & $\frac{0.063}{0.018}=3.5$ & 8.16 \\
\hline (3) & $\frac{3 s-2 p}{5 s-2 p}$ & $\rightarrow$ & $\frac{0.063}{0.0127}=4.96$ & 6.63 \\
\hline (4) & $\frac{3 s-2 p}{5 s-3 p}$ & $\rightarrow$ & $\frac{0.063}{0.0085}=7.41$ & 17.6 \\
\hline (5) & $\frac{3 s-2 p}{5 s-4 p}$ & $\rightarrow$ & $\frac{0.063}{0.0065}=9.69$ & 38.1 \\
\hline (6) & $\frac{3 s-2 p}{6 s-2 p}$ & $\rightarrow$ & $\frac{0.063}{0.0073}=8.63$ & 13.4 \\
\hline (7) & $\frac{3 s-2 p}{6 s-3 p}$ & $\rightarrow$ & $\frac{0.063}{0.0051}=12.35$ & 4.16 \\
\hline (8) & $\frac{3 s-2 p}{6 s-4 p}$ & $\rightarrow$ & $\frac{0.063}{0.0035}=18.0$ & 70.1 \\
\hline (9) & $\frac{3 s-2 p}{6 s-5 p}$ & $\rightarrow$ & $\frac{0.063}{0.0017}=37.1$ & 129 \\
\hline (10) & $\frac{4 s-2 p}{4 s-3 p}$ & $\rightarrow$ & $\frac{0.025}{0.018}=1.39$ & 2.86 \\
\hline (11) & $\frac{4 s-2 p}{5 s-2 p}$ & $\rightarrow$ & $\frac{0.025}{0.0127}=1.97$ & 2.32 \\
\hline (12) & $\frac{4 s-2 p}{5 s-3 p}$ & $\rightarrow$ & $\frac{0.025}{0.0085}=2.94$ & 6.17 \\
\hline (13) & $\frac{4 s-2 p}{5 s-4 p}$ & $\rightarrow$ & $\frac{0.025}{0.0065}=3.85$ & 13.3 \\
\hline (14) & $\frac{4 s-2 p}{6 s-2 p}$ & $\rightarrow$ & $\frac{0.025}{0.0073}=3.42$ & 4.68 \\
\hline (15) & $\frac{4 s-3 p}{5 s-2 p}$ & $\rightarrow$ & $\frac{0.018}{0.0127}=1.42$ & 0.81 \\
\hline (16) & $\frac{4 s-3 p}{5 s-3 p}$ & $\rightarrow$ & $\frac{0.018}{0.0085}=2.11$ & 2.16 \\
\hline (17) & $\frac{4 s-3 p}{5 s-4 p}$ & $\rightarrow$ & $\frac{0.018}{0.0065}=2.77$ & 4.67 \\
\hline (18) & $\frac{4 s-3 p}{6 s-2 p}$ & $\rightarrow$ & $\frac{0.018}{0.0073}=2.47$ & 1.64 \\
\hline
\end{tabular}
the intensity ratios calculated in Table 3. 


\section{Continued}

(19)

$$
\frac{4 s-3 p}{6 s-3 p} \quad \rightarrow \quad \frac{0.018}{0.0051}=3.53
$$

(20)

$\frac{4 s-3 p}{6 s-4 p} \rightarrow \frac{0.018}{0.0035}=5.14$

8.59

(21)

$\frac{4 s-3 p}{6 s-5 p} \quad \rightarrow \quad \frac{0.018}{0.0017}=10.6$

15.8

(22)

$\frac{5 s-2 p}{5 s-3 p} \quad \rightarrow \quad \frac{0.0127}{0.0085}=1.49$;

2.66

(23)

$\frac{5 s-2 p}{5 s-4 p} \quad \rightarrow \quad \frac{0.0127}{0.0065}=1.95$

5.74

(24)

$\frac{5 s-2 p}{6 s-2 p} \quad \rightarrow \quad \frac{0.0127}{0.0073}=1.74 ;$

2.02

(25)

$$
\frac{5 s-2 p}{6 s-3 p} \quad \rightarrow \quad \frac{0.0127}{0.0051}=2.49 ;
$$

(26)

$$
\frac{5 s-2 p}{6 s-4 p} \rightarrow \frac{0.0127}{0.0035}=3.63
$$

$$
\frac{5 s-2 p}{6 s-5 p} \quad \rightarrow \quad \frac{0.0127}{0.0017}=7.47 \text {; }
$$

(28)

$$
\frac{5 s-3 p}{5 s-4 p} \quad \rightarrow \quad \frac{0.0085}{0.0065}=1.31
$$

$$
\frac{5 s-3 p}{6 s-2 p} \quad \rightarrow \quad \frac{0.0085}{0.0073}=1.16
$$

$$
\frac{5 s-3 p}{6 s-3 p} \quad \rightarrow \quad \frac{0.0085}{0.0051}=1.67
$$

$$
\frac{5 s-3 p}{6 s-4 p} \rightarrow \frac{0.0085}{0.0035}=2.43 ;
$$

$$
\frac{5 s-3 p}{6 s-5 p} \rightarrow \frac{0.0085}{0.0017}=5.0
$$

$$
\frac{5 s-4 p}{6 s-2 p} \rightarrow \frac{0.0065}{0.0073}=0.89 ;
$$

$$
\frac{5 s-4 p}{6 s-3 p} \quad \rightarrow \quad \frac{0.0065}{0.0051}=1.27
$$

$$
\frac{5 s-4 p}{6 s-4 p} \rightarrow \frac{0.0065}{0.0035}=1.86
$$

$$
\frac{5 s-4 p}{6 s-5 p} \quad \rightarrow \quad \frac{0.0065}{0.0017}=3.82 ;
$$

$$
\frac{6 s-2 p}{6 s-3 p} \quad \rightarrow \quad \frac{0.0073}{0.0051}=1.43
$$

$$
\frac{6 s-2 p}{6 s-4 p} \quad \rightarrow \quad \frac{0.0073}{0.0035}=2.09
$$

$$
\frac{6 s-2 p}{6 s-5 p} \quad \rightarrow \quad \frac{0.0073}{0.0017}=4.29
$$

$$
\frac{6 s-3 p}{6 s-4 p} \rightarrow \frac{0.0051}{0.0035}=1.46
$$

$\frac{6 s-3 p}{6 s-5 p} \rightarrow \frac{0.0051}{0.0017}=3.0$ 
If the larger of the ratios from the semiclassical and quantum-mechanical pair is considered in each examined case, all other ratios of this kind—beyond of the case (61) presented in (24)—do not exceed 1.5. In average this is evidently a much better agreement between the semiclassical and quantum-mechanical theory than attained for transitions $n^{\prime} p-n^{\prime \prime} s \quad\left(n^{\prime}>n^{\prime \prime}\right)$ considered in [8].

A different situation is represented, however, by the semiclassical data collected in Table 3 compared with the quantum-mechanical results in Table 4. An agreement between these sets of the data is evidently poorer than attained in case of Table 1 and Table 2 of the present paper, as well as for the data collected in [8]. In 16 cases [(2), (4), (5), (7)-(10), (12), (13), (23), (25)-(27), (33), (38) and (39)] the quantum-mechanical ratio divided by the semiclassical one (or vice versa, so the resulted value $>1$ is always obtained) exceeds 2 . Maximal ratios are given by cases (5) and (8) for which the results are respectively

$$
\frac{\text { semiclassical ratio }}{\text { quantum-mechanical ratio }}=\frac{38.1}{9.69}=3.93 ; \quad \frac{70.1}{18.0}=3.89 \text {; }
$$

see Table 4.

Because of a qualitatively different reference between the angular momenta in the beginning and end quantum states entering transitions examined respectively in Table 1 and Table 3, it can be supposed that not only the energy levels but also the values of the angular momenta can influence the ratio of the semiclassical intensities. At present we have no insight into the angular-momentum contribution to the semiclassical results for the intensity relations.

\section{Quantum Aspect of the Joule-Lenz Law Referred to the Bohr Correspondence Principle}

A well-known reference to the correspodence principle concerns the energy spectrum of the Bohr hydrogen atom [11] [12].

Let us examine a difference which exists between the frequency of the electron motion about the atomic nucleus in state $n$ represented by

$$
v_{n}=\frac{1}{T_{n}}=\frac{2 R_{H}}{n^{3}},
$$

or a similar circulation frequency in state $m$ represented by

$$
v_{m}=\frac{1}{T_{m}}=\frac{2 R_{H}}{m^{3}},
$$

and the frequency associated with the electron transition from level $m$ to level $n$

$$
v_{q}=R_{H}\left(\frac{1}{n^{2}}-\frac{1}{m^{2}}\right)=R_{H} \frac{(m-n)(m+n)}{m^{2} n^{2}}
$$

which corresponds to the electromagnetic wave emitted in effect of the transition. The $T_{n}$ and $T_{m}$ in (26) and (27) are the time periods of the circular motion about the nucleus and

$$
R_{H}=\frac{2 \pi^{2} m_{e} e^{4}}{h^{3}}=\frac{m_{e} e^{4}}{4 \pi \hbar^{3}}
$$

is the Rydberg constant.

If

$$
m-n=\Delta n=1
$$

and $m$ as well and $n$ are large numbers, we obtain from (28) the relation

$$
v_{q} \cong \frac{2 R_{H}}{m^{3}} \approx \frac{2 R_{H}}{n^{3}}
$$

Since the energy of the atom say in state $n$ is

$$
E_{n}=-\frac{R_{H} h}{n^{2}}
$$


the action integral in the same state is

$$
J_{n}=\oint \boldsymbol{p}_{n} \mathrm{~d} \boldsymbol{r}_{n}=2 \pi p_{n} r_{n}=2 \pi m v_{n} r_{n}=2 \pi n \hbar=n h,
$$

because of the electron velocity

$$
v_{n}=\frac{e^{2}}{n \hbar}
$$

and the orbit radius

$$
r_{n}=\frac{n^{2} \hbar^{2}}{m_{e} e^{2}} .
$$

Both quantities $E_{n}$ and $J_{n}$ become coupled together by the relation

$$
v_{n}=\frac{\mathrm{d} E_{n}}{\mathrm{~d} J_{n}}=\frac{\mathrm{d}}{\mathrm{d}(n h)}\left(-\frac{R_{H} h}{n^{2}}\right)=\frac{2 R_{H}}{n^{3}}
$$

which gives a result identical with (26).

For low $m$ and $n$, and $\Delta n$ given in (30) the result (36) does not hold because in this case

$$
v_{q}=\frac{\Delta E}{\Delta J} \Delta n=\frac{\Delta E}{\Delta J}=\frac{\Delta E}{h}
$$

which is evidently different than (36) because of the validity of (28). The idea of the correspondence principle represented by the formula (36) was that it works only on condition $m, n \gg 1$.

The importance of the Joule-Lenz law comes from the fact that—contrary to the correspondence principleits quantum aspects can be applied for low energy transitions concerning also the low energy states labelled by small $n$ [8]. This is due to the property that a good accuracy of the formula

$$
\Delta E_{n} \Delta t_{n}=h,
$$

in which

$$
\Delta E_{n}=E_{n+1}-E_{n}
$$

and

$$
\Delta t_{n}=t_{n+1}-t_{n},
$$

does exist also for small $n$.

In fact the interval (40) is the time difference between two situations represented respectively by the neighbouring quantum values of the energy, $E_{n+1}$ and $E_{n}$. The result that

$$
\Delta t_{n}=T_{n}
$$

in only an approximate one, though it becomes well satisfied already at low $n$. In practical calculations, especially for the low-energy transitions, the formula (41) can be neglected but only (38) applied. This is done in the present and the former paper [8]. Since (38) does hold solely for the neighbouring energy states any time interval

$$
\Delta t=t_{n+q}-t_{n}
$$

for $q>1$ can be transformed into a sum of the step intervals

$$
\Delta t=t_{n+q}-t_{n+q-1}+t_{n+q-1}-t_{n+q-2}+\cdots+t_{n+1}-t_{n}
$$

with the formula (38) applied separately to each step entering (43); see [8].

It seems useful to be noted that the orbital velocity $v_{n}$ in (34) can be obtained as a drift velocity [13]

$$
v_{d}=c \frac{\left|\boldsymbol{E}_{n}\right|}{\left|\boldsymbol{B}_{n}\right|}
$$

of the electron in the presence of the electric 


$$
\left|\boldsymbol{E}_{n}\right|=\frac{e}{r_{n}^{2}}=\frac{e^{5} m_{e}^{2}}{n^{4} \hbar^{4}}
$$

and magnetic

$$
\left|\boldsymbol{B}_{n}\right|=\frac{e^{3} m_{e}^{2} c}{n^{3} \hbar^{3}}
$$

field; cf. here [14] for $\left|\boldsymbol{B}_{n}\right|$. In consequence from (44)-(46) we obtain

$$
v_{d}=c \frac{e^{5} m_{e}^{2}}{n^{4} \hbar^{4}} \frac{n^{3} \hbar^{3}}{e^{3} m_{e}^{2} c}=\frac{e^{2}}{n \hbar}
$$

which is identical with (34).

\section{Summary}

The time seems to be a not favourite parameter for the quantum physicists. In fact, the quantum events occupy so short intervals of time that their accurate measurement seems to be hardly possible. A similar difficulty concerns a precise definition of the beginning or end time of the quantum process. In effect the time as a measurable observable enters quite seldom the quantum-theoretical analysis or an empirical observation.

The aim of the present and a former paper [8] was to demonstrate-by applying the analysis of quantum properties deduced from the Joule-Lenz law-that the time interval available for a single energy emission between two neighbouring quantum states can be next useful in description of more extended emission processes.

This led to situation that the patterns of the emission intensity between the states of the hydrogen atom calculated with the aid of quantum mechanics could be compared with the semiclassical patterns of intensity data obtained from the time intervals which are characteristic for the electron transitions between the energy levels in the atom. It should be added that all transitions entering calculations have been selected according to the wellknown rules of quantum mechanics concerning the electron angular momentum.

An evident similarity of the semiclassical and quantum-mechanical patterns of intensity has been attained. A better agreement between the data of the both methods occurs in case of transitions when a larger angular momentum is associated with the beginning state of emission, i.e. the state having also a larger energy, for example the transition is $n^{\prime} d-n^{\prime \prime} p$ and $n^{\prime}>n^{\prime \prime}$.

\section{References}

[1] Planck, M. (1910) Acht Vorlesungen ueber Theoretische Physik. S. Hirzel, Leipzig.

[2] Einstein, A. (1917) Physikalische Zeitschrift, 18, 121.

[3] Kuhn, T.S. (1987) Black-Body Theory and the Quantum Discontinuity, 1894-1912. University of Chicago Press, Chicago.

[4] Bethe, H. (1933) Handbuch der Physik. In: Geiger, H. and Scheel, K., Eds., Vol. 24, Part 1, Springer, Berlin.

[5] Condon, E.U. and Shortley, G.H. (1970) The Theory of Atomic Spectra. Cambridge University Press, Cambridge.

[6] Olszewski, S. (2015) Journal of Modern Physics, 6, 1277-1288; Olszewski, S. (2016) Reviews in Theoretical Science, 4, 336-352. http://dx.doi.org/10.4236/jmp.2015.69133; http://dx.doi.org/10.1166/rits.2016.1066

[7] Olszewski, S. (2016) Journal of Modern Physics, 7, 162-174. http://dx.doi.org/10.4236/jmp.2016.71018

[8] Olszewski, S. (2016) Journal of Modern Physics, 7, 827-851. http://dx.doi.org/10.4236/jmp.2016.78076

[9] Lass, H. (1960) Vector and Tensor Analysis. McGraw-Hill, New York.

[10] Kuhn, H.G. (1962) Atomic Spectra. Academic Press, New York.

[11] Sommerfeld, A. (1931) Atombau and Spektrallinien. Vol. 1, 5th Edition, Braunschweig.

[12] Slater, J.C. (1960) Quantum Theory of the Atomic Structure. Vol. 1, McGraw-Hill, New York.

[13] Matveev, A.N. (1964) Electrodynamics and the Theory of Relativity. Izd. Wyzszaja Szkola, Moscow. (In Russian)

[14] Olszewski, S. (2015) Journal of Modern Physics, 6, 2202-2210. 